\author{
MITSUBISHI ELECTRIC RESEARCH LABORATORIES \\ http://www.merl.com
}

\title{
Unified Spectral Efficiency Analysis of Cellular Systems with Channel-Aware Schedulers
}

\author{
Wu, J.; Mehta, N.B.; Molisch, A.F.; Zhang, J.
}

TR2011-078 December 2011

\begin{abstract}
Spectral efficiency is a key characteristic of cellular communications systems, as it quantifies how well the scarce spectrum resource is utilized. It is influenced by the scheduling algorithm as well as the signal and interference statistics, which in turn depend on the propagation characteristics. In this paper we derive analytical expressions for the short-term and long-term channel-averaged spectral efficiencies of the round robin, greedy Max-SINR, and proportional fair schedulers, which are popular and cover a wide range of system performance and fairness trade-offs. A unified spectral efficiency analysis is developed to highlight the differences among these schedulers. Unlike previous work in the literature, the analysis is notably different in the following aspects: (i) it does not assume the co-channel interferer to be identically distributed, as is typical in realistic cellular layouts, (ii) it avoids the loose spectral efficiency bounds used in the literature, which only considered the worst case and best case locations of identical co-channel interferer, (iii) it explicitly includes the effect of multi-tier interferer in the cellular layout and uses a more accurate model for handling the total co-channel interference, and (iv) it captures the impact of using small modulation constellation sizes, which are typical of second and third-generation cellular standards. The analytical results are verified using extensive Monte Carlo simulations.
\end{abstract}

\section{IEEE Transactions on Communications}

\footnotetext{
This work may not be copied or reproduced in whole or in part for any commercial purpose. Permission to copy in whole or in part without payment of fee is granted for nonprofit educational and research purposes provided that all such whole or partial copies include the following: a notice that such copying is by permission of Mitsubishi Electric Research Laboratories, Inc.; an acknowledgment of the authors and individual contributions to the work; and all applicable portions of the copyright notice. Copying, reproduction, or republishing for any other purpose shall require a license with payment of fee to Mitsubishi Electric Research Laboratories, Inc. All rights reserved.
} 



\title{
Unified Spectral Efficiency Analysis of Cellular Systems with Channel-Aware Schedulers
}

\author{
Jingxian $\mathrm{Wu}^{*}$, Member, IEEE, Neelesh B. Mehta ${ }^{\dagger}$, Senior Member, IEEE, \\ Andreas F. Molisch ${ }^{\star}$, Fellow, IEEE, and Jin Zhang ${ }^{\ddagger}$, Fellow, IEEE
}

\begin{abstract}
Spectral efficiency is a key characteristic of cellular communications systems, as it quantifies how well the scarce spectrum resource is utilized. It is influenced by the scheduling algorithm as well as the signal and interference statistics, which in turn depend on the propagation characteristics. In this paper we derive analytical expressions for the short-term and long-term channel-averaged spectral efficiencies of the round robin, greedy Max-SINR, and proportional fair schedulers, which are popular and cover a wide range of system performance and fairness trade-offs. A unified spectral efficiency analysis is developed to highlight the differences among these schedulers. Unlike previous work in the literature, the analysis is notably different in the following aspects: (i) it does not assume the co-channel interferers to be identically distributed, as is typical in realistic cellular layouts, (ii) it avoids the loose spectral efficiency bounds used in the literature, which only considered the worst case and best case locations of identical co-channel interferers, (iii) it explicitly includes the effect of multi-tier interferers in the cellular layout and uses a more accurate model for handling the total cochannel interference, and (iv) it captures the impact of using small modulation constellation sizes, which are typical of secondand third-generation cellular standards. The analytical results are verified using extensive Monte Carlo simulations.
\end{abstract}

Index Terms - spectral efficiency, Max-SINR, proportional fair, round robin, scheduler, lognormal, fading, shadowing

\section{INTRODUCTION}

Cellular communication systems strive to achieve high spectral efficiencies in order to efficiently utilize the scarce and expensive spectrum resource and deliver higher data rates to users within the limited bandwidth. This needs to be done in the presence of effects such as large-scale fading, which arises due to shadowing, and small-scale fading, which arises due to multipath components, and in an environment in which aggressive frequency reuse leads to severe co-channel interference (CCI) from neighboring cells [1]. To achieve the desired high spectral efficiencies, third generation and beyond systems employ advanced techniques such as link adaptation and channel-aware multi-user scheduling [2], [3].

Part of this paper was previously presented at the 2005 IEEE Global Telecommunication Conference (GLOBECOM'05), St. Louis, MO, U.S.A., and the 2007 IEEE International Conference on Communications (ICC'07), Glasgow, Scotland.

${ }^{*} \mathrm{~J}$. Wu is with the Dept. of Electrical Engineering, University of Arkansas, Fayetteville, AR 72701, USA. ${ }^{\dagger}$ N. B. Mehta is with the Dept. of Electrical Communication Eng. at the Indian Institute of Science (IISc), Bangalore, India. ${ }^{\star}$ A. F. Molisch is with the Electrical Engineering Dept. at the University of Southern California, CA, USA. $\ddagger$ J. Zhang is with the Mitsubishi Electric Research Labs (MERL), 201 Broadway, Cambridge, MA 02139, USA. (Emails: wuj@uark.edu, nbmehta@ece.iisc.ernet.in, andreas.molisch@ieee.org, jzhang@merl.com)

The results presented in this paper are based on the initial research work performed by all the authors at MERL.
Spectral efficiency, which measures the average data rate per unit bandwidth per cell, is an important measure for quantitatively evaluating the effectiveness of cellular systems. It plays an important role in system planning, performance analysis, and optimization. It captures the fundamental tradeoff between the limited spectrum resource and the growing demands of broadband service, and is critical in the planning and design of cellular systems. For example, decreasing frequency reuse distance leads to stronger CCI, which results in a smaller spectral efficiency for each individual user and, thus, a smaller user-averaged spectral efficiency. However, more aggressive frequency reuse means more bandwidth per unit area; thus, more users can be supported in a unit area, and this might improve the overall spectral efficiency.

An analytical characterization of the spectral efficiency is quite involved. The presence of CCI and the competition for radio resources at the scheduler make it very difficult to extrapolate the multi-cell multi-user system-level performance results from single transmitter single receiver link-level results, especially in the presence of fading and shadowing. Therefore, most performance evaluations thus far have been simulation studies [2], [4], [5], with many of these using very standardspecific models. On the other hand, many of the analytical models for average spectral efficiency (ASE) use simplifying assumptions that limit the accuracy and applicability of their results. For example, in [6], an excellent analytical framework is developed to quantify the ASE of interference-limited cellular systems in the presence of Rayleigh fading and/or lognormal shadowing. However, its analysis, and also the analyses in [7], [8], assume that the mean power of all the interferers is identical. Consequently, the results derived become performance bounds that assume that all users are at the best-case or worst-case locations for interference - a situation that does not occur in practical settings. The bounds turn out to be quite loose for small reuse distances, at which next generation systems will operate. Such an approach also prevents the inclusion of second-tier interference and sectorization in the analysis. Though smaller than first-tier interference, the effect of second-tier interference on spectral efficiency is not negligible. For example, its inclusion is mandatory for systemlevel simulations in the $3 \mathrm{GPP}$ and WiMAX standardization frameworks [9].

To capture the performance of the entire cellular system, it is also critical that the analysis captures the dynamic spectrum sharing among multiple competing users. Most of the aforementioned works [6], [10] implicitly assume in their analyses that all users are given equal resources within a cell, which 
is valid only for a round-robin scheduling policy. However, third generation cellular systems and beyond use channelaware schedulers that exploit multi-user diversity to achieve spectral efficiency gains. Therefore, it is of great importance to incorporate different schedulers into the analysis and do so in a unified manner that enables them to be compared. While an opportunistic RR scheduler [11], and a proportional fair scheduler [12], [13], [14], which achieves a balance between fairness and system throughput, have been analyzed, this has been done only for a single-cell system with Rayleigh fading and no CCI [11], [13], [14]. While [15] did consider the effect of multi-tier interference and different schedulers, its analysis assumes that the throughput directly equals the signal to interference ratio (SIR) at the receiver; this makes its results applicable only to systems in which the SIR at each mobile station is very small. While [16] derived the throughput of different schedulers, it is only applicable to a single-cell system without any CCI. Thus, there is a great need to develop an analysis of a multi-cell framework that incorporates both interference and the non-linear relationship of throughput with signal-to-interference-plus-noise-ratio (SINR).

Channel variation in a cellular environment occurs due to both Rayleigh fading and lognormal shadowing, which operate on vastly different times scales. While the 'short-term' Rayleigh fading typically varies on the order of milliseconds, the 'long-term' shadowing changes over tens of seconds or more depending on the user mobility. Thus, averaging the spectral efficiency over short-term Rayleigh fading and longterm shadowing provides different insights into the system behavior. Averaging over the fading (conditioned on the shadowing) reveals the impact of fading on system behavior over a short period of time. The long-term behavior of cellular system can be understood by averaging the instantaneous spectral efficiency over the lognormal shadowing as well. Doing so provides guidelines for the design of cellular layouts and the identification of service coverage areas. Consequently, this dual timescale approach has also been followed in [6].

This paper presents a general analysis of the spectral efficiency achievable by cellular systems that factors in the interactions of all the above mentioned effects, including scheduling, interference, and wireless channel propagation. Specifically, it makes the following contributions. It derives general expressions for the spectral efficiency of (possibly sectorized) cellular systems with channel-aware schedulers that operate with non-identical co-channel interferers. The individual and combined impacts of both small-scale Rayleigh fading and large-scale lognormal shadowing are incorporated in the analysis. The efficiency achieved with the following broad range of schedulers is analyzed: (i) the Max-SINR scheduler, which fully exploits the multi-user diversity but is unfair [16], (ii) the Round-Robin scheduler, which is fair but channelunaware, and (iii) the Proportional Fair (PF) scheduler, which features the tradeoff between fairness and throughput. The new unified analytical results serve to highlight their performance differences. The analysis also accounts for the impact of small modulation constellation sizes, and is valid for both an interference-limited system that employs aggressive frequency reuse and operates closer to peak capability; and a noise- or coverage-limited system that employs a large frequency reuse distance.

The rest of this paper is organized as follows. The cellular system model is introduced in Section II. The short-term fading-averaged and long-term composite shadowing-averaged spectral efficiencies of the various schedulers are derived in Section III and Section IV, respectively. Numerical examples in Section V are followed by our conclusions in Section VI.

\section{PRELIMINARIES}

\section{A. Cellular System Downlink Model}

Consider a cellular system with $N$ users per cell or sector. In the downlink, each user copes with CCI from $M$ neighboring base stations (BSs). The received signal at a user, say $n$, which has been selected by the scheduler for downlink transmission can be written as:

$$
r_{n}=h_{n 0} x_{n 0}+\sum_{m=1}^{M} h_{n m} x_{n m}+z_{n},
$$

where $x_{n 0}$ is the desired signal transmitted by the serving BS (chosen without loss of generality to have index 0), $x_{n m}$ is the signal transmitted by an interfering $\mathrm{BS} m$, and $z_{n}$ is additive white Gaussian noise. The powers of $x_{n 0}$ and $x_{n m}$ are normalized to unity. The channels are assumed to be flatfading; we assume that both BSs and mobile stations (MSs) have only a single antenna.

The channel coefficient, $h_{n m}$, is the instantaneous gain of the channel between the $n$-th MS and the $m$-th BS. It can be written as $h_{n m}=\sqrt{\alpha_{n m}} g_{n m}$, where $\alpha_{n m}$ includes the effects of transmission power, pathloss, shadowing, and directional antenna gain at BS and MS. The small-scale fading term $g_{n m}$ is modeled as a zero-mean, unit-variance complex Gaussian random variable (RV). The lognormal probability density function (pdf) of $\alpha_{n m}$ is given by

$$
f_{\alpha_{n m}}(x)=\frac{\xi}{\sqrt{2 \pi} \sigma_{n m} x} \exp \left[-\frac{\left(\xi \log _{e} x-\mu_{n m}\right)^{2}}{2 \sigma_{n m}^{2}}\right], \quad x \geq 0,
$$

where $\mu_{n m}$ and $\sigma_{n m}^{2}$ are the $\mathrm{dB}$ mean and variance, respectively, of the Gaussian RV $10 \log _{10} \alpha_{n m}$, and $\xi=10 / \log _{e} 10$. Specifically, $\mu_{n m}=P_{m}-L_{0}-10 p \log _{10}\left(d_{n m} / d_{0}\right)-A\left(\theta_{n m}\right)-$ $B\left(\theta_{n m}\right)$, where $P_{m}$ is the transmission power (in dBW) of the $m$-th BS, $L_{0}$ is the pathloss (in $\mathrm{dB}$ ) at a reference distance $d_{0}$ from the BS, $d_{n m}$ the distance of the $n$-th MS from the $m$-th BS, $p$ is the pathloss exponent, $A\left(\theta_{n m}\right)$ and $B\left(\theta_{n m}\right)$ are the antenna gains (in $\mathrm{dB}$ ) of the BS and MS, respectively, and $\theta_{n m}$ is the angle between the antenna boresight and the line of sight form the $n$-th MS to the $m$-th BS. For example, in [20], $A(\theta)$ is specified as

$$
A(\theta)=-\min \left[12\left(\frac{\theta}{\theta_{0}}\right)^{2}, A_{0}\right],-180^{\circ} \leq \theta \leq 180^{\circ},
$$

where $\theta_{0}=70^{\circ}$ and $A_{0}=20 \mathrm{~dB}$ for a 3 -sector cell, and $\theta_{0}=35^{\circ}$ and $A_{0}=23 \mathrm{~dB}$ for a 6 -sector cell. For a 1 -sector cell, $A(\theta)=0 \mathrm{~dB} . B\left(\theta_{n m}\right)$ is usually set to unity. For cell with multiple sectors, the available bandwidth is equally divided among the sectors. 


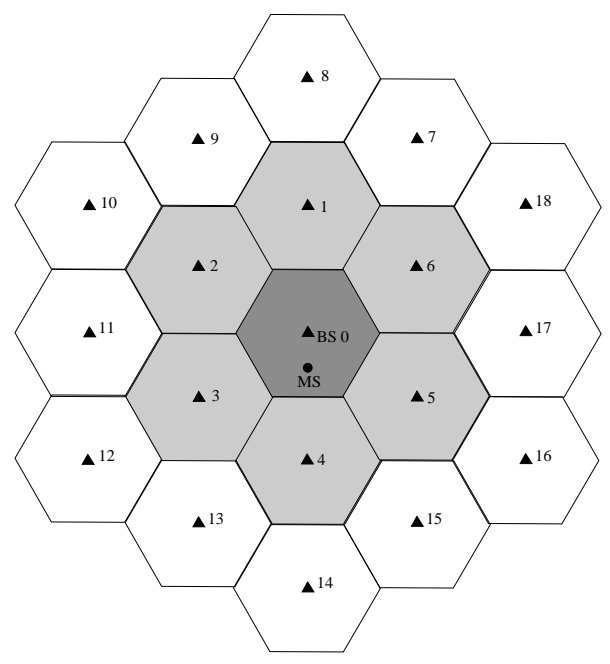

Fig. 1. The geometric layout of a mobile radio system with 19 cells.

The number of interferers depends on the geometric layout of the cellular system and sectorization. For example, for the hexagonal layout shown in Figure 1, when only firsttier interferers are considered, we have $M=6$ without sectorization, $M=2$ for 3 sectors per cell, and $M=1$ for 6 sectors per cell [21]. When the second-tier interferers are also considered, the corresponding values are $M=18, M=7$, and $M=4$. In actual systems, the cell layout depends strongly on the geography and morphology of the environment, and the pathloss has to be determined from empirical or ray-tracing data. We stress that even in this case, the closed-form equations developed in the next sections are applicable.

The instantaneous SINR, $\gamma_{n}$, at the receiver of the scheduled user, $n$, is given by

$$
\gamma_{n}=\frac{\alpha_{n 0}\left|g_{n 0}\right|^{2}}{\sum_{m=1}^{M} \alpha_{n m}\left|g_{n m}\right|^{2}+1 / \rho},
$$

where $\rho$ is the fading-averaged signal-to-noise-ratio (SNR). It is assumed that only one user in each sector is scheduled at any instant. We assume that neither dirty-paper coding at the BS nor multi-user detection at the MS are used.

\section{B. Spectral Efficiency}

Spectral efficiency captures the highest data throughput per unit bandwidth achievable by the entire cellular system under the limitations imposed by the system model assumptions. We, therefore, use the Shannon capacity formula to measure throughput [6], as it is the maximum throughput the channel can reliably support given a certain SINR constraint.

The impact of a limited modulation constellation size is modeled by means of a cap, $C_{\max }$, on the achievable throughput per unit bandwidth as follows:

$$
C\left(\gamma_{n}\right)=\left\{\begin{array}{ll}
\log _{2}\left(1+\gamma_{n}\right), & \gamma_{n} \leq \gamma_{T} \\
C_{\max }, & \gamma_{n}>\gamma_{T}
\end{array},\right.
$$

where $T$ is the allowed maximum modulation constellation size and $C_{\max }=\log _{2}\left(1+\gamma_{T}\right)$. When no constraint is placed on the constellation size, we have $C_{\max }=\infty$.
The instantaneous SINR, $\gamma_{n}$, varies with respect to time due to the time-varying nature of the fading channel. Link adaptation techniques, such as adaptive modulation and coding (AMC), can be adopted to adjust the data rate based on the channel condition indicated by the SINR value. The formula in (5) models the case where capacity-achieving error-free codes are used and the transmitter adapts its transmission rates on a continuous scale. Spectral efficiencies of systems with practical adaptive AMC schemes or uncoded systems can be obtained by scaling the SINR in (5) with a factor less than 1 [4]; the analytical methods presented in this paper can be extended to this case.

The schedulers operate in real time, i.e., at a rate fast enough to adapt to the small-scale Rayleigh fading variations. Thus, the formula in (5) gives the expression for the instantaneous spectral efficiency, which changes as the fading channels of the multiple users vary with time. The planning of cellular layout and the identification of service coverage area necessitate the evaluation of channel-averaged spectral efficiency.

The channel-averaged spectral efficiency for the $n$-th user can be written as

$$
C_{n}=\int_{0}^{\infty} C(\gamma) f(\gamma) d \gamma
$$

where the function, $f(\gamma)$, depends on the specific averaging operation discussed earlier. For short-term Rayleigh fadingaveraged spectral efficiency, $f(\gamma)=f_{\gamma_{n} \mid \alpha}(\gamma)$, which is the conditional pdf of the SINR, $\gamma_{n}$, conditioned on the shadowing coefficients, $\alpha_{n m}$. On the other hand, for long-term (Rayleigh fading and lognormal shadowing) averaged spectral efficiency, $f(\gamma)=f_{\gamma_{n}}(\gamma)$, with $f_{\gamma_{n}}(\gamma)$ being the unconditional pdf of the SINR that includes both Rayleigh fading and lognormal shadowing.

Substituting (5) into (6) and simplifying lead to the following alternative spectral efficiency expression:

$$
C_{n}=\log _{2}(e) \int_{0}^{\gamma_{T}} \frac{1}{1+\gamma}[1-F(\gamma)] d \gamma,
$$

where $F(\gamma)=F_{\gamma_{n} \mid \alpha}(\gamma)$ is the conditional cumulative distribution function (CDF) of $\gamma_{n}$ for short-term fading averaged analysis, and $F(\gamma)=F_{\gamma_{n}}(\gamma)$ is the corresponding unconditional CDF for fading and shadowing-averaged analysis.

The system-level spectral efficiency of cellular systems employing the fair round robin scheduler, the greedy MaxSINR scheduler, and the proportional fair scheduler will be analyzed in the next two sections under a unified framework.

\section{ShORT-Term RAYleigh FAding-Averaged SPECTRAL EFFICIENCY}

We first consider the impact of short-term fading on systemlevel performance by deriving fading-averaged expressions for the spectral efficiency. If large-scale fading is present, such an averaging leads to spectral efficiency expressions that are conditioned on the shadowing. 
Lemma 1: The pdf of the SINR of the $n$-th user in a Rayleigh fading environment can be written as

$$
f_{\gamma_{n} \mid \alpha}(\gamma)=-\frac{\partial}{\partial \gamma}\left[\prod_{m=1}^{M}\left(1+\frac{\alpha_{n m}}{\alpha_{n 0}} \gamma\right)^{-1} \exp \left(-\frac{\gamma}{\rho} \frac{1}{\alpha_{n 0}}\right)\right]
$$

Proof: The proof is given in Appendix A.

The differential form in (8) shall come in handy later on. It also leads to the following corollary about the CDF of $\gamma_{n}$, which is useful for outage analyses [8].

Corollary 1: The CDF of the SINR, $\gamma_{n}$, of user $n$ in a Rayleigh fading environment is

$$
F_{\gamma_{n} \mid \alpha}(\gamma)=1-\exp \left(-\frac{\gamma}{\rho} \frac{1}{\alpha_{n 0}}\right) \prod_{m=1}^{M}\left(1+\frac{\alpha_{n m}}{\alpha_{n 0}} \gamma\right)^{-1}
$$

\section{A. Spectral Efficiency of Round-Robin scheduler}

In a system with the RR scheduler, once a user is served by the BS, it is not served again until all the other users in the system have been served. The RR scheduler has the same average spectral efficiency as a random scheduler, which schedules all users with the same probability and does not take into account the channel states of the users. We state, without proof, the following simple Lemma on the average spectral efficiency of the RR scheduler.

Lemma 2: The average spectral efficiency of a cellular system with $N$ users and an RR scheduler is

$$
C_{\mathrm{RR}}=\frac{1}{N} \sum_{n=1}^{N} C_{n}
$$

where $C_{n}$ is the average spectral efficiency of the $n$-th user: $C_{n}=\int_{0}^{\infty} C(\gamma) f_{\gamma_{n} \mid \alpha}(\gamma) d \gamma$.

Thus, $C_{\mathrm{RR}}$ is simply the average of all the individual users' average spectral efficiencies. The above formula was assumed throughout in [6]-[8], [10], which is why their ASE analyses apply only to the RR scheduler.

1) Single User Average Spectral Efficiency Analysis:

Lemma 3: The Rayleigh fading-averaged spectral efficiency of user $n$ in closed-form is

$$
\begin{gathered}
C_{n}=\log _{2}(e) \sum_{l=1}^{L_{n}} \sum_{i=1}^{m_{n}^{(l)}} \frac{\beta_{i}^{(l)}}{(i-1) !}\left[\frac{\alpha_{n 0}}{\alpha_{n}^{(l)}}\right]^{m_{n}^{(l)}} \hat{\gamma}_{n 0}^{i-m_{n}^{(l)}} e^{\frac{1}{\hat{\gamma}_{n}^{(l)}}} \\
\times\left[\Gamma\left(i-m_{n}^{(l)}, \frac{1}{\hat{\gamma}_{n}^{(l)}}\right)-\Gamma\left(i-m_{n}^{(l)}, \frac{1}{\hat{\gamma}_{n}^{(l)}}+\frac{2^{C_{\max }}-1}{\hat{\gamma}_{n 0}}\right)\right],
\end{gathered}
$$

where $\Gamma(k, x)$ is the incomplete Gamma function [22]. Here, the $(M+1)$ variables, $\left\{\alpha_{n m}\right\}_{m=0}^{M}$, are partitioned into $L_{n}$ subsets, such that each subset contains all and only the variables with the same value $\alpha_{n}^{(l)} . m_{n}^{(l)}$ denotes the cardinality of the $l$-th subset (where $M+1=\sum_{l=1}^{L_{n}} m_{n}^{(l)}$ ). $C_{\max }$ is the maximum rate allowed by the system, $\hat{\gamma}_{n}^{(l)}=\rho \alpha_{n}^{(l)}$, $\hat{\gamma}_{n 0}=\rho \alpha_{n 0}$, and the coefficient $\beta_{i}^{(l)}$ is

$$
\beta_{i}^{(l)}=\left.\frac{\partial^{i-1}}{\partial \gamma^{i-1}}\left[\left(1+\frac{\alpha_{n}^{(l)}}{\alpha_{n 0}} \gamma\right)^{m_{n}^{(l)} M} \prod_{m=0}^{M}\left(1+\frac{\alpha_{n m}}{\alpha_{n 0}} \gamma\right)^{-1}\right]\right|_{\gamma=-\frac{\alpha_{n 0}}{\alpha^{(l)}}} .
$$

Proof: The proof is given in Appendix B.

In a practical setting, the means $\left\{\alpha_{n m}\right\}_{m=1}^{M}$ are distinct due to different path-losses, shadowing, and antenna gains. $C_{n}$ then simplifies to

$$
\begin{aligned}
C_{n} & =\log _{2}(e) \sum_{m=0}^{M} \frac{\alpha_{n 0}}{\alpha_{n m}}\left(\prod_{\substack{i=0 \\
i \neq m}}^{M} \frac{\alpha_{n m}}{\alpha_{n m}-\alpha_{n i}}\right) e^{\frac{1}{\hat{\gamma}_{n m}}} \\
& \times\left[\Gamma\left(0, \frac{1}{\hat{\gamma}_{n m}}\right)-\Gamma\left(0, \frac{1}{\hat{\gamma}_{n m}}+\frac{2^{C_{\max }}-1}{\hat{\gamma}_{n 0}}\right)\right] .
\end{aligned}
$$

The overall spectral efficiency of the RR scheduler can now be computed from the single user values (of Lemma 3) using Lemma 2. The result in Lemma 3 accounts for the effects of both noise and CCI. For systems with aggressive frequency reuse that operate close to peak capability, the statistical properties of the SINR are dominated by CCI such that the effect of noise is negligible, i.e., $\rho \rightarrow \infty$. Such systems are interference-limited. When $\rho \rightarrow \infty$, the CDF $F_{\gamma_{n} \mid \alpha}(\gamma)=1-\prod_{m=1}^{M}\left(1+\frac{\alpha_{n m}}{\alpha_{n 0}} \gamma\right)^{-1}$. Substituting $F_{\gamma_{n} \mid \alpha}(\gamma)$ into (7), and performing partial fraction expansion we have the interference-limited spectral efficiency given as follows.

Corollary 2: The Rayleigh fading-averaged spectral efficiency of a given user in an interference-limited environment can be expressed as

$$
\begin{aligned}
& C_{n}= \log _{2}(e) \sum_{l=1}^{L_{n}} \sum_{i=1}^{m_{n}^{(l)}-1} \frac{\beta_{i}^{(l)}}{(i-1) !}\left[\frac{\alpha_{n 0}}{\alpha_{n}^{(l)}}\right]^{m_{n}^{(l)}} \frac{1}{m_{n}^{(l)}-i} \\
& \times\left\{\left[\frac{\alpha_{n 0}}{\alpha_{n}^{(l)}}\right]^{i-m_{n}^{(l)}}-\left[\frac{\alpha_{n 0}}{\alpha_{n}^{(l)}}+2^{C_{\max }}-1\right]^{i-m_{n}^{(l)}}\right\} \\
&+\log _{2}(e) \sum_{l=1}^{L_{n}} \frac{\beta_{m_{n}^{(l)}}^{(l)}}{\left(m_{n}^{(l)}-1\right) !}\left[\frac{\alpha_{n 0}}{\alpha_{n}^{(l)}}\right]^{m_{n}^{(l)}} \ln \left[1+\frac{\alpha_{n}^{(l)}}{\alpha_{n 0}}\left(2^{C_{\max }}-1\right)\right] .
\end{aligned}
$$

On the other hand, when the reuse distance is large such that the CCI is negligible compared to noise, the system can be modeled as a noise-limited system. Setting $M=0$ in (11) or (13), leads to the following corollary about the spectral efficiency of a noise-limited system.

Corollary 3: The Rayleigh fading-averaged spectral efficiency of a given user in a noise-limited environment (or an isolated cell system) can be expressed as

$$
C_{n}=\log _{2}(e) e^{\frac{1}{\hat{\gamma}_{n 0}}}\left[\Gamma\left(0, \frac{1}{\hat{\gamma}_{n 0}}\right)-\Gamma\left(0, \frac{2^{C_{\max }}-1}{\hat{\gamma}_{n 0}}\right)\right] .
$$

Setting $C_{\max }=\infty$ in the above equation and simplifying leads to the result obtained previously in [23, eqn. (12)] for noise-limited systems with unlimited constellation sizes. 


\section{B. Spectral Efficiency of Max-SINR Scheduler}

While the RR scheduler ensures fairness among users, it does so at the expense of a reduced overall system throughput. The Max-SINR scheduler, on the other hand, serves the mobile station with the highest SINR among all the users. Let $\gamma_{\max }=$ $\max \left\{\gamma_{1}, \gamma_{2}, \cdots, \gamma_{N}\right\}$ denote the maximum SINR among all users at any instant. The average spectral efficiency of a system with the Max-SINR scheduler is [c.f. (6)]

$$
C_{\text {MSINR }}=\log _{2}(e) \int_{0}^{\gamma_{T}} \frac{1}{1+\gamma}\left(1-F_{\gamma_{\max }}(\gamma)\right) d \gamma,
$$

where $F_{\gamma_{\max }}(\gamma)$ is the CDF of $\gamma_{\max }$.

1) Statistics of $\gamma_{\max }$ : To evaluate $C_{\text {MSINR }}$ in (15), we first need the CDF of $\gamma_{\max }$. This is derived below.

Lemma 4: The CDF of $\gamma_{\max }$ is given by

$F_{\gamma_{\max }}(\gamma)=1+\sum_{n=1}^{N}(-1)^{n} \sum_{k=1}^{\left(\begin{array}{c}N \\ n\end{array}\right)} \exp \left(-\delta_{n k} \gamma\right) \prod_{i \in \mathcal{S}_{k}(N, n)} \prod_{m=1}^{M}\left(1+\lambda_{i m} \gamma\right)^{-1}$

where $\delta_{n k}=\sum_{i \in \mathcal{S}_{k}(N, n)} \frac{1}{\gamma_{i 0} \alpha_{i 0}}, \quad \lambda_{i m}=\alpha_{i m} / \alpha_{i 0}$, and $\mathcal{S}_{k}(N, n)$ is an $n$-element subset of the index set $\{1,2, \cdots, N\}$. The number of possible subsets is $\left(\begin{array}{l}N \\ n\end{array}\right)$, and $k$ indexes all of these subsets.

Proof: The proof is given in Appendix C.

To facilitate the analysis, define the following function:

$$
\Phi_{n, k}(\gamma)=\frac{1}{1+\gamma} \prod_{i \in \mathcal{S}_{k}(N, n)} \prod_{m=1}^{M}\left(1+\lambda_{i m} \gamma\right)^{-1} .
$$

Furthermore, the product terms of the form $\left(1+\lambda_{i m} \gamma\right)^{-1}$ in $\Phi_{n, k}(x)$ are partitioned into $L(n, k)$ subsets, such that each subset (of size $m_{l}(n, k)$ ) contains all the terms with the same value of $\lambda_{i m}$. With the above book-keeping notation in place, we have the following theorem about the average spectral efficiency of the Max-SINR scheduler.

Theorem 1: The average spectral efficiency expression for a cellular system with $N$ users and the Max-SINR scheduler in a Rayleigh fading environment is given by

$$
\begin{gathered}
C_{\text {MSINR }}=\log _{2}(e) \sum_{n=0}^{N}(-1)^{n-1} \sum_{k=1}^{\left(\begin{array}{c}
N \\
n
\end{array}\right)} \sum_{l=1}^{L(n, k)} \lambda_{l}(n, k)^{-m_{l}(n, k)} \\
\times \sum_{i=1}^{m_{l}(n, k)} \frac{\beta_{i}^{(l)}(n, k)}{(i-1) !} \delta_{n k}^{m_{l}(n, k)-i} \exp \left[\frac{\delta_{n k}}{\lambda_{l}(n, k)}\right] \\
\times\left\{\begin{array}{l}
\Gamma\left[i-m_{l}(n, k), \frac{\delta_{n k}}{\lambda_{l}(n, k)}\right] \\
-\Gamma\left[i-m_{l}(n, k), \frac{\delta_{n k}}{\lambda_{l}(n, k)}+\delta_{n k}\left(2^{C_{\max }}-1\right)\right]
\end{array}\right\} .
\end{gathered}
$$

Here, $\delta_{n k}$ is defined in Lemma 4 , and the coefficients $\beta_{i}^{(l)}(n, k)$, for $i=1, \cdots, m_{l}(n, k)$, are given by

$\beta_{i}^{(l)}(n, k)=\left.\frac{\partial^{i-1}}{\partial \gamma^{i-1}}\left[\left(1+\lambda_{l}(n, k) \gamma\right)^{m_{l}(n, k)} \Phi_{n, k}(\gamma)\right]\right|_{\gamma=-}$.

Proof: The proof is given in Appendix D.

The above result simplifies considerably when the interferers are non-identical.

In an interference-limited environment, the integrand in (15) can be written as $\sum_{n=1}^{N}(-1)^{n-1} \sum_{k=1}^{\left(\begin{array}{c}N \\ n\end{array}\right)} \Phi_{n, k}(\gamma)$ when $\rho \rightarrow$ $\infty$. Performing partial fraction expansion of $\Phi_{n, k}(\gamma)$ and using it in (15), we get the following interference-limited spectral efficiency of the Max-SINR scheduler.

Corollary 4: The Rayleigh fading-averaged spectral efficiency of an interference-limited cellular system with the MaxSINR scheduler is given in (18) at the top of the next page.

In a noise-limited environment (or an isolated cell system), the CDF of $\gamma_{\max }$ can be simplified to: $F_{\gamma_{\max }}(\gamma)=$ $1+\sum_{n=1}^{N}(-1)^{n} \sum_{k=1}^{\left(\begin{array}{c}N \\ n\end{array}\right)} \exp \left(-\delta_{n k} \gamma\right)$. Substituting it into (15) leads to the following corollary.

Corollary 5: The Rayleigh fading-averaged spectral efficiency of a noise-limited cellular system with the Max-SINR scheduler is

$$
\begin{aligned}
C_{\mathrm{MSNR}}=\log _{2}(e) \sum_{n=1}^{N}(-1)^{n+1} \sum_{k=1}^{\left(\begin{array}{c}
N \\
n
\end{array}\right)} \exp \left(\delta_{n k}\right) \\
\times\left[\Gamma\left(0, \delta_{n k}\right)-\Gamma\left(0, \delta_{n k}\left(2^{C_{\max }}-1\right)\right)\right] .
\end{aligned}
$$

\section{Spectral Efficiency of Proportional Fair Scheduler}

The PF scheduler balances fairness and the use of multi-user diversity, to enhance cell throughput [12]. The PF scheduler makes the base station transmit to a user who has the largest value of the following normalized metric [13] $\varphi_{n}=\frac{\gamma_{n}}{\bar{\gamma}_{n}}$, where $\bar{\gamma}_{n}=E\left(\gamma_{n}\right)$ is the average SINR of the $n$-th user. The value of $\bar{\gamma}_{n}$ can be obtained at the scheduler through a time averaging mechanism. It should be noted that alternate metrics are possible for the PF scheduler. For example, the rate-based metric, $C\left(\gamma_{n}\right) / \bar{C}\left(\gamma_{n}\right)$, with $C\left(\gamma_{n}\right)$ being the instantaneous spectral efficiency and $\bar{C}\left(\gamma_{n}\right)$ a moving window averaged spectral efficiency, is used in [2], [14] (and the references therein). The SINR-based metric and the original rate-based metric share the same important characteristics, and allocate almost the same portion of time slots to each user [13].

The $n$-th user is scheduled if $\varphi_{n}>\max _{k,(k \neq n)}\left(\varphi_{k}\right)$. Define $\varphi_{\max , n}=\max _{k,(k \neq n)}\left(\varphi_{k}\right)$ as the largest metric of all other users except user $n$. User $n$ is scheduled if $\gamma_{n}>\bar{\gamma}_{n} \cdot \varphi_{\max , n}$. Thus, the cell spectral efficiency of the PF scheduler is [13]

$$
C_{\mathrm{PF}}=\sum_{n=1}^{N} E\left[C\left(\gamma_{n}\right) \mid \gamma_{n}>\bar{\gamma}_{n} \varphi_{\max , n}\right] P\left(\gamma_{n}>\bar{\gamma}_{n} \varphi_{\max , n}\right),
$$

where $C\left(\gamma_{n}\right)$ is defined in (5), and the expectation is performed over the small-scale fading of all users.

Equation (20) can be expanded to $C_{\mathrm{PF}}=$ $\sum_{n=1}^{N} \int_{0}^{\infty} C(x) f_{\gamma_{n} \mid \alpha}(x) F_{\varphi_{\max , n}}\left(\frac{x}{\bar{\gamma}_{n}}\right) d x$, where $F_{\varphi_{\max , n}}(x)$ is the $\mathrm{CDF}$ of the $\mathrm{RV} \varphi_{\max , n}$, and takes the form $F_{\varphi_{\max , n}}(x)=\prod_{\substack{k=1 \\ k \neq n)}}^{N} F_{\varphi_{k}}(x)=\prod_{\substack{k=1 \\ k \neq n}}^{N} F_{\gamma_{k} \mid \alpha}\left(\bar{\gamma}_{k} x\right)$. Combining the above equations leads to

$$
C_{\mathrm{PF}}=\sum_{n=1}^{N} \int_{0}^{\infty} C(x) f_{\gamma_{n} \mid \alpha}(x)\left[\prod_{\substack{k=1 \\ k \neq n}}^{N} F_{\gamma_{k} \mid \alpha}\left(\frac{\bar{\gamma}_{k}}{\bar{\gamma}_{n}} x\right)\right] d x
$$




$$
\begin{aligned}
C_{\mathrm{MSINR}}=\log _{2}(e) \sum_{n=0}^{N}(-1)^{n-1} & \sum_{k=1}^{\left(\begin{array}{c}
N \\
n
\end{array}\right)} \sum_{l=1}^{L(n, k)} \lambda_{l}(n, k)^{-m_{l}(n, k)}\left\{\sum _ { i = 1 } ^ { m _ { l } ( n , k ) - 1 } \frac { \beta _ { i } ^ { ( l ) } ( n , k ) } { ( i - 1 ) ! } \frac { 1 } { m _ { l } ( n , k ) - i } \left[\lambda_{l}(n, k)^{m_{l}(n, k)-i}\right.\right. \\
& \left.\left.+\left(\lambda_{l}(n, k)^{-1}+2^{C_{\max }}-1\right)^{i-m_{l}(n, k)}\right]+\frac{\beta_{m_{l}(n, k)}^{(l)}(n, k)}{\left(m_{l}(n, k)-1\right) !} \ln \left[1+\lambda_{l}(n, k)\left(2^{C_{\max }}-1\right)\right]\right\}
\end{aligned}
$$

Further analytical simplification is difficult due to the product of various CDFs and a $\log$ function in $C(x)$. The spectral efficiency, therefore, needs to be evaluated numerically by substituting (5), (8), and (9) into (21). However, closedform expressions can be obtained, as shown below, if we assume that the scheduling metrics, $\left\{\varphi_{n}\right\}_{n=1}^{N}$, are i. i. d. This approximation makes intuitive sense because the fairness of the PF scheduler comes from the scheduling metrics being approximately identically distributed. In addition, the scheduling metrics are obtained by normalizing the SINR with respect to their respective mean values, which takes care of the different MS locations. The accuracy of this i. i. d. approximation is validated by simulation results in Section V.

Theorem 2: The short-term Rayleigh fading-averaged spectral efficiency of the PF scheduler with the assumption of i. i. d. scheduling metrics is given by

$$
\begin{gathered}
C_{\mathrm{PF}}=\frac{\log _{2}(e)}{N} \sum_{n=0}^{N} \sum_{k=1}^{N}(-1)^{k-1}\left(\begin{array}{c}
N \\
k
\end{array}\right) \sum_{l=1}^{L_{n}}\left(\frac{\alpha_{n 0}}{\alpha_{n l}}\right)^{\hat{m}_{n}^{(l)}} \\
\times \sum_{i=1}^{\hat{m}_{n}^{(l)}} \hat{\beta}_{i}^{(l)}(n, k)\left(\frac{\hat{\gamma}_{n 0}}{k}\right)^{i-\hat{m}_{n}^{(l)}} e^{\frac{k}{\hat{\gamma}_{n}^{(l)}}} \\
\times\left[\Gamma\left(i-\hat{m}_{n}^{(l)}, \frac{k}{\hat{\gamma}_{n}^{(l)}}\right)-\Gamma\left(i-\hat{m}_{n}^{(l)}, \frac{k}{\hat{\gamma}_{n l}}+\frac{k\left(2^{C_{\max }}-1\right)}{\hat{\gamma}_{n 0}}\right)\right],
\end{gathered}
$$

where, as in Lemma 3 , the $M+1$ variables $\left\{\alpha_{n m}\right\}_{m=0}^{M}$ are partitioned into $L_{n}$ subsets, with each subset containing all and only the variables with the same value $\alpha_{n}^{(l)}$. Without loss of generality, let $\alpha_{n 0}$ be in the first subset, i. e., $\alpha_{n 0}=\alpha_{n}^{(1)}$. Then $\hat{m}_{n}^{(l)}=1+k\left(m_{n}^{(l)}-1\right)$ if $l=1$, and $\hat{m}_{n}^{(l)}=k m_{n}^{(l)}$, for $1<l \leq L_{n}$, where $m_{n}^{(l)}$ is the cardinality of the $l$-th subset as defined in Lemma 3 . The coefficient $\hat{\beta}_{i}^{(l)}$ is

$$
\begin{gathered}
\hat{\beta}_{i}^{(l)}=\left.\frac{1}{(i-1) ! \partial \gamma^{i-1}}\left[\left(1+\frac{\alpha_{n}^{(l)}}{\alpha_{n 0}} \gamma\right)^{\hat{m}_{n}^{(l)} M} \prod_{m=0}^{i-1}\left(1+\frac{\alpha_{n m}}{\alpha_{n 0}} \gamma\right)^{-1}\right]\right|_{\gamma=-\frac{\alpha_{n 0}}{\alpha_{n}^{(l)}}} . \\
\text { Proof: The proof is given in Appendix E. }
\end{gathered}
$$

As before, the average spectral efficiency expression of an interference-limited system can be obtained by letting $\rho \rightarrow \infty$, and the integrand of the spectral efficiency expression can be expanded as

$$
\frac{\left[1-F_{\gamma_{n}}^{N}(x)\right]}{1+x}=\sum_{k=1}^{N}(-1)^{k-1}\left(\begin{array}{l}
N \\
k
\end{array}\right) \prod_{l=1}^{L_{n}}\left(1+\frac{\alpha_{n}^{(l)}}{\alpha_{n 0}} x\right)^{-\hat{m}_{n}^{(l)}} .
$$

Performing partial fraction expansion of the product term in the above expressionleads to the following corollary.
Corollary 6: Assuming that the scheduling metrics are i. i. d., the Rayleigh fading-averaged spectral efficiency of an interference-limited cellular system with the PF scheduler is

$$
\begin{gathered}
C_{\mathrm{PF}}=\frac{\log _{2}(e)}{N} \sum_{n=0}^{N} \sum_{k=1}^{N}(-1)^{k-1}\left(\begin{array}{c}
N \\
k
\end{array}\right) \sum_{l=1}^{L_{n}}\left(\frac{\alpha_{n 0}}{\alpha_{n l}}\right)^{\hat{m}_{n}^{(l)}} \\
\times\left\{\sum_{i=1}^{\hat{m}_{n}^{(l)}-1} \frac{\hat{\beta}_{i}^{(l)}(n, k)}{\hat{m}_{n}^{(l)}-i}\left[\left(\frac{\alpha_{n 0}}{\alpha_{n l}}\right)^{i-\hat{m}_{n}^{(l)}}+\left(\frac{\alpha_{n 0}}{\alpha_{n l}}+2^{C_{\max }}-1\right)^{i-\hat{m}_{n}^{(l)}}\right]\right. \\
\left.+\hat{\beta}_{\hat{m}_{n}^{(l)}}^{(l)}(n, k) \ln \left[1+\frac{\alpha_{n l}}{\alpha_{n 0}}\left(2^{C_{\max }}-1\right)\right]\right\} .
\end{gathered}
$$

In a noise-limited environment, the average spectral efficiency is obtained by setting $m=0$, and the result is stated as follows.

Corollary 7: Assuming that the scheduling metrics are i. i. d., the Rayleigh fading-averaged spectral efficiency of a noise-limited cellular system with the PF scheduler is

$$
\begin{aligned}
C_{\mathrm{PF}}=\frac{\log _{2}(e)}{N} & \sum_{n=0}^{N} \sum_{k=1}^{N}\left(\begin{array}{c}
N \\
k
\end{array}\right)(-1)^{k-1} e^{\frac{k}{\hat{\gamma}_{n 0}}} \\
& \times\left[\Gamma\left(0, \frac{k}{\hat{\gamma}_{n 0}}\right)-\Gamma\left(0, \frac{k 2^{C_{\mathrm{max}}}}{\hat{\gamma}_{n 0}}\right)\right] .
\end{aligned}
$$

No i. i. d. metric approximation is necessary for the special case in which the SINRs of all users are i. i. d, i.e., $\bar{\gamma}_{k}=\bar{\gamma}_{n}$, $\forall n \neq k$. In such a case, the spectral efficiency expression of the PF scheduler simplifies to

$$
C_{\mathrm{PF} \text {,i.i. }}=\int_{0}^{\infty} C(x) d\left[\prod_{k=1}^{N} F_{\gamma_{k} \mid \alpha}(x)\right]=C_{\mathrm{MSINR}} .
$$

The above result is intuitively obvious since the the Max-SINR scheduler is fair when all the users have the same average SINR as all the users are served for the same average time duration.

\section{A Unified Spectral Efficiency Expression for All Schedulers}

Based on the analysis so far, we can write a unified expression for the spectral efficiency of RR, Max-SINR, and PF schedulers. This provides further insights about the performance differences among the different schedulers.

From (7) and (15), the spectral efficiency of all the three schedulers is given by

$$
C=\log _{2}(e) \frac{1}{N} \sum_{n=1}^{N} \int_{0}^{\gamma_{T}} \frac{1}{1+x} G_{n}(x) d x
$$


where

$$
G_{n}(x)= \begin{cases}1-F_{\gamma_{n} \mid \alpha}(x), & \text { RR scheduler, } \\ 1-F_{\gamma_{n} \mid \alpha}^{N}(x), & \text { PF scheduler, } \\ 1-F_{\gamma_{\max }}(x), & \text { Max-SINR scheduler. }\end{cases}
$$

The following intuitively obvious corollary then follows.

Corollary 8: The spectral efficiencies of the RR, PF, and Max-SINR schedulers are related by the following inequality:

$$
C_{\mathrm{RR}} \leq C_{\mathrm{PF}} \leq C_{\mathrm{MSINR}}
$$

where $C_{\mathrm{RR}}=C_{\mathrm{PF}}$ if and only if the SINRs $\left\{\gamma_{n}\right\}_{n=1}^{N}$ are deterministic, and $C_{\mathrm{PF}}=C_{\mathrm{MSINR}}$ if and only if the SINRs $\left\{\gamma_{n}\right\}_{n=1}^{N}$ are i. i. d.

Proof: The proof is given in Appendix F.

The above analysis is based on the assumption that the scheduler has perfect knowledge of the channel state information. It is expected that channel estimation error in practical systems will negatively affect the performance of the channelaware Max-SINR and PF schedulers, but will have negligible impact on the channel-unaware RR scheduler.

\section{LONG-TERM SPECTRAL EFFICIENCY ANALYSIS UNDER COMPOSITE CHANNELS}

We now investigate the spectral efficiency when both smallscale Rayleigh fading and long-term lognormal shadowing are considered. For analytical tractability, we limit our analysis to interference-limited systems in which the noise is negligible [4], [6]-[8], which is the case when the cellular systems are operating closer to peak capability. Define the SIR as

$$
\gamma_{n}=\frac{S_{n}}{\sum_{m=1}^{M} I_{n m}}
$$

where $S_{n}=\alpha_{n 0}\left|g_{n 0}\right|^{2}$ is the received desired signal component power, and $I_{n m}=\alpha_{n m}\left|g_{n m}\right|^{2}$ is the power of the co-channel interference from the $m$-th BS.

As before, we first analyze the statistics of the SIR of a single user. Due to the combined effects of shadowing, and fading, the received signal power, $S_{n}$, and interference power, $I_{n m}$, follow a composite Rayleigh-lognormal distribution, for which no closed-form pdf formula is available [24]. However, it has been shown in [26] that the SIR, $\gamma_{n}$, can be accurately approximated by a lognormal $\mathrm{RV}, \tilde{\gamma}_{n}$, whose parameters are determined using an efficient and flexible moment generating function (MGF) matching method [17]. The CDF of the lognormally distributed SIR, $\tilde{\gamma}_{n}$, can then be written as

$$
F_{\tilde{\gamma}_{n}}(\gamma)=1-Q\left(\frac{10 \log _{10} \gamma-\mu_{\tilde{\gamma}_{n}}}{\sigma_{\tilde{\gamma}_{n}}}\right)
$$

where $Q(x)=\frac{1}{\sqrt{2 \pi}} \int_{x}^{+\infty} \exp \left(-\frac{y^{2}}{2}\right) d y$ is the Gaussian-Q function and $\mu_{\tilde{S}_{n}}$ and $\sigma_{\tilde{S}_{n}}^{2}$ are the $\mathrm{dB}$ moments of the lognormal RV $\tilde{\gamma}_{n}$. The values of $\mu_{\tilde{S}_{n}}$ and $\sigma_{\tilde{s}_{n}}^{2}$ can be obtained with the method described in [26].

\section{A. Spectral Efficiency of the Round-Robin Scheduler}

The average spectral efficiency, $\tilde{C}_{\mathrm{RR}}$, with a RR scheduler is now obtained by combining (7), (10), and (29), as

$$
\tilde{C}_{\mathrm{RR}}=\frac{\log _{2} e}{N} \sum_{n=1}^{N} \int_{0}^{\gamma_{T}} \frac{1}{1+\gamma} Q\left(\frac{\xi \log _{e} \gamma-\mu_{\tilde{\gamma}_{n}}}{\sigma_{\tilde{\gamma}_{n}}}\right) d \gamma .
$$

Since the Gaussian-Q function is itself an integral, the average spectral efficiency expression in (30) requires a twofold integration. This can be simplified by using the following accurate and recent approximation that expresses the GaussianQ function [19] in terms of elementary functions only:

$$
\hat{Q}(x)=\frac{\left(1-e^{-A x}\right) e^{-x^{2}}}{B \sqrt{\pi} x}, \text { for } x>0,
$$

where $A=1.98$ and $B=1.135$ are obtained through an optimization described in [19]. Using $Q(x)=1-Q(-x)$, for $x<0$, we can simplify the representation of $\tilde{C}_{\mathrm{RR}}$ by combining (30) and (31). The final expression is a single finite integral with only elementary functions in its integrand.

Alternate approaches have been used in the literature to address the difficulty in analytically simplifying (30). In [6], upper and lower bounds of the function $\log _{2}(1+\gamma)$ are used. However, the bounds are loose for small reuse distances. The approximation $\log _{2}(1+\gamma) \approx \gamma$ was used in [15]. However, this approximation works only for small values of $\gamma$. Using the approximation of (31) leads to a more accurate characterization of the SIR over a wider range of its values.

\section{B. Spectral Efficiency of the Max-SIR Scheduler}

The Max-SIR scheduler serves the mobile station with the highest SIR. Let $\tilde{\gamma}_{\max }=\max \left\{\tilde{\gamma}_{1}, \tilde{\gamma}_{2}, \cdots, \tilde{\gamma}_{N}\right\}$ denote the maximum SIR among all users at any instant. From (29), the CDF of $\gamma_{\max }$ can be written as

$$
F_{\tilde{\gamma}_{\max }}(\gamma)=\prod_{n=1}^{N}\left[1-Q\left(\frac{\xi \log _{e} \gamma-\mu_{\tilde{\gamma}_{n}}}{\sigma_{\tilde{\gamma}_{n}}}\right)\right]
$$

where the equality follows because the SIRs $\left\{\tilde{\gamma}_{n}\right\}_{n=1}^{N}$ are independent. After substituting (32) into (15), the spectral efficiency of the Max-SIR scheduler upon averaging over composite fading is

$$
\left.\widetilde{C}_{\mathrm{MSIR}}=\log _{2}(e) \int_{0}^{\gamma_{T} 1} \frac{1+\gamma}{1+\prod_{n=1}^{N}}\left[1-Q\left(\frac{\xi \log _{e} \gamma-\mu_{\tilde{\gamma}_{n}}}{\sigma_{\tilde{\gamma}_{n}}}\right)\right]\right\} d \gamma .
$$

The above expression can be simplified by using (31).

\section{Spectral Efficiency of the Proportional Fair Scheduler}

The PF scheduler serves the mobile station with the highest scheduling metric $\tilde{\varphi}_{n}=\tilde{\gamma}_{n} / \overline{\tilde{\gamma}}_{n}$, where $\overline{\tilde{\gamma}}_{n}=E\left(\tilde{\gamma}_{n}\right)$ is the shadowing and fading-averaged mean of the SIR $\tilde{\gamma}_{n}$. Assume, as before, that the metrics $\left\{\tilde{\varphi}_{n}\right\}_{n=1}^{N}$ are identically distributed. This results in the following expression for the spectral efficiency of the PF scheduler, which is obtained by combining (25) and (29):

$\tilde{C}_{\mathrm{PF}}=\frac{\log _{2}(e)}{N} \sum_{n=1}^{N} \sum_{k=1}^{N}\left(\begin{array}{c}N \\ k\end{array}\right) \int_{0}^{\gamma_{T}} \frac{(-1)^{k-1}}{1+\gamma} Q^{k}\left(\frac{\xi \log _{e} \gamma-\mu_{\tilde{\gamma}_{n}}}{\sigma_{\tilde{\gamma}_{n}}}\right) d \gamma$. 
Applying the Gaussian-Q function approximation in the above equation yields an expression with a single integral.

It should be noted that the above spectral efficiency expressions are also applicable to systems operating in a noiselimited composite fading-shadowing environment or in an environment with only lognormal shadowing and no (or minimal) Rayleigh fading. In both cases, the only change in the above analysis is the recomputation of $\mu_{\tilde{\gamma}_{n}}$ and $\sigma_{\tilde{\gamma}_{n}}^{2}$.

\section{NUMERICAL EXAMPLES}

We now compare the analytical results with Monte Carlo simulations and quantify the effects of the main system parameters. A representative hexagonal cellular layout, shown in Figure 1, with a reuse factor of 1 and up to two tiers of interfering BSs is used. The pathloss exponent is assumed to be 3.7 [1]. The $\mathrm{dB}$ standard deviation of all lognormal RVs is $\sigma=8$. Unless otherwise specified, the $k$-th user is placed at a distance of $\frac{k}{N} R$ from its serving $\mathrm{BS}$, where $R$ is the cell radius, and at an azimuth of $\frac{2 k \pi}{N}$. Such a user placement scheme can help understand the effect of different interference statistics at different user locations. Each simulation point is generated with 50,000 realizations of small-scale and large-scale fading.

\section{A. Small-scale Rayleigh Fading-Averaged Spectral Efficiency}

Figure 2 plots the spectral efficiencies of the Max-SINR, PF, and RR schedulers for different numbers of MSs, $N$, in the cell. The SNR at the cell corner is $\mu=10 \mathrm{~dB}$. As expected, the Max-SINR Scheduler increases most as $N$ increases, since it benefits the most from multiuser diversity, followed by PF scheduler and RR scheduler. Notice that the simulation and analytical results agree very well for all three schedulers. The analytical results of the PF scheduler are obtained based on the approximation that the scheduling metrics are i. i. d, while the simulations are performed without such an assumption. The excellent match between the simulation and analytical results verifies the accuracy of this approximation.

The spectral efficiencies of the RR scheduler and the MaxSINR scheduler in 1-sector, 3-sector, and 6-sector cells are plotted in Figure 3 as a function of the SNR at the cell corner.

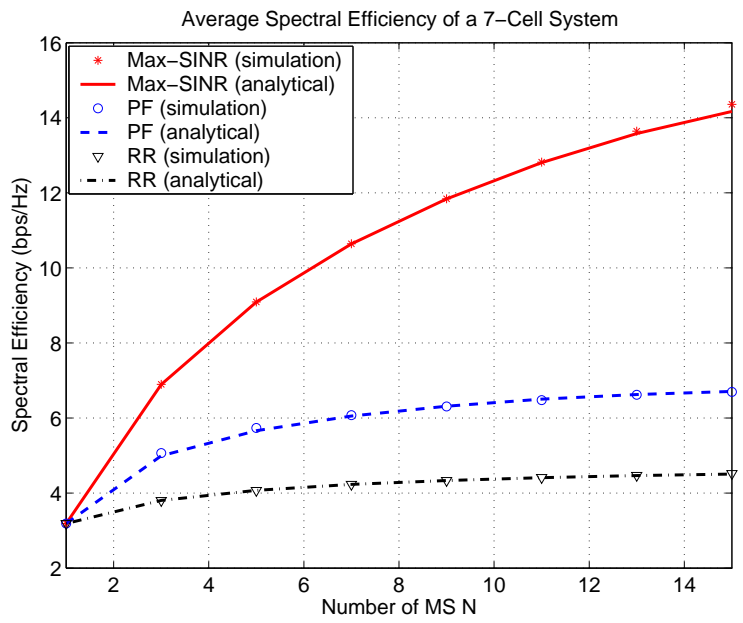

Fig. 2. Short-term Rayleigh fading-averaged spectral efficiencies of systems with various schedulers.

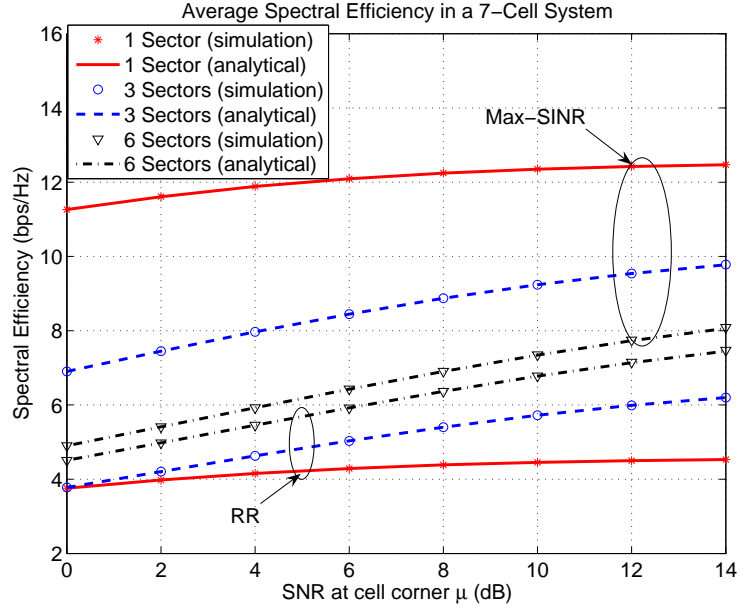

Fig. 3. Short-term Rayleigh fading-averaged spectral efficiencies of the MaxSINR scheduler and the RR scheduler with different number of sectors as a function of cell corner SNR.

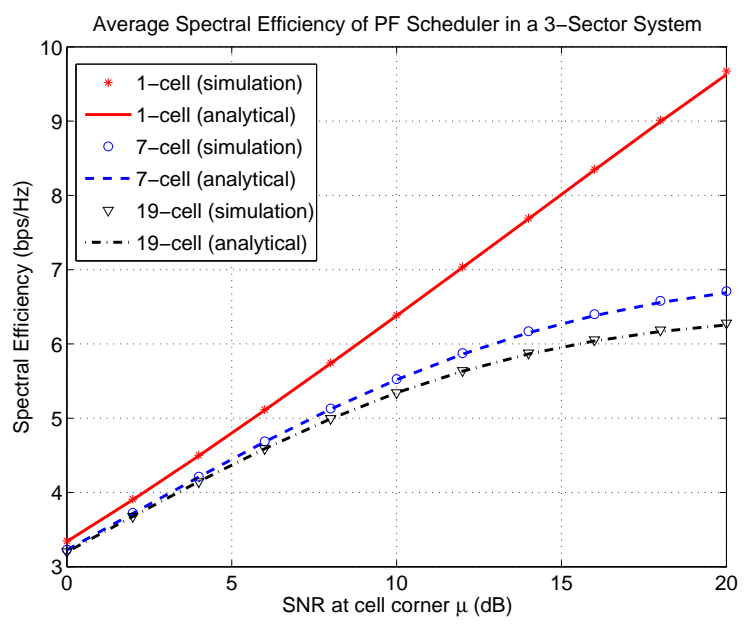

Fig. 4. Short-term Rayleigh fading-averaged spectral efficiencies of the PF scheduler with different number of CCIs.

The number of users in the center cell is 10 . The total system bandwidth is evenly divided among sectors. One user in each sector is scheduled at any moment. It is interesting to note that sectorization has opposite effects on Max-SINR and RR schedulers. Sectorization benefits RR scheduler by reducing the number of CCIs. On the other hand, the performance of the Max-SINR scheduler suffers from sectorization. This performance degradation is because the bandwidth must be shared by the best users in each sector, whereas the entire bandwidth is allocated to the best user in a one-sector system. The reduction of CCI due to sectorization is not enough to compensate for the capacity loss incurred by spectrum sharing among sectors for the Max-SINR scheduler.

Figure 4 plots the spectral efficiency of the PF scheduler in the absence of CCI, with only first-tier interferers, and with both first- and second-tier interferers. Such second-tier interferers can be easily included in our analysis since it is not constrained by the assumption that they are identically distributed. There are 15 users uniformly distributed in the center cell. Each cell has 3 sectors. It can be seen that CCI 


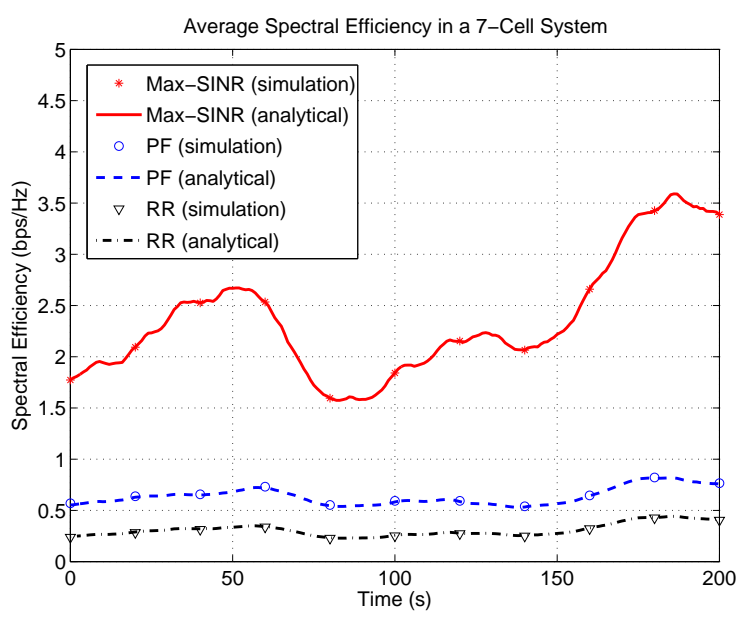

Fig. 5. Short-term spectral efficiency of the system with faster time-varying Rayleigh fading and slower time-varying lognormal shadowing as a function of time when averaged over a window of duration $5 \mathrm{sec}$.

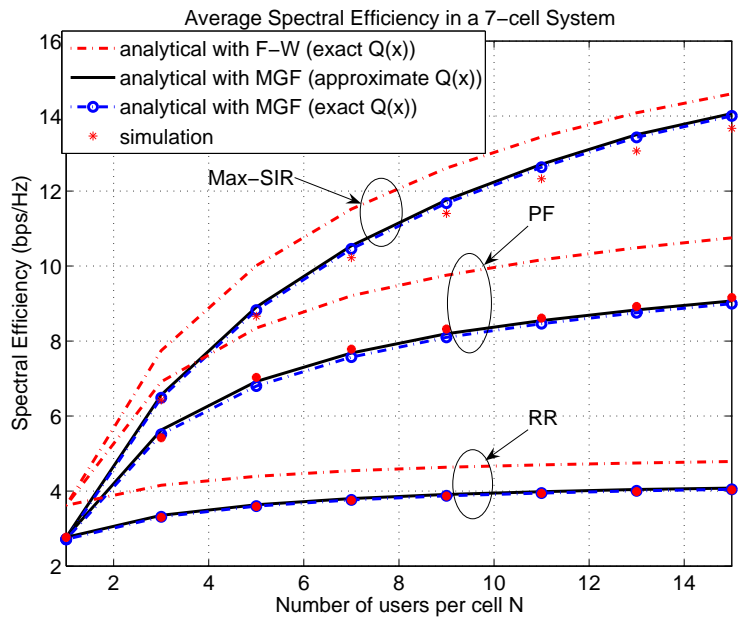

Fig. 6. Composite channels: Comparison of spectral efficiency results from analysis (using different lognormal sum approximation methods) and simulations for RR, PF, and Max-SIR schedulers.

has a significant impact on the spectral efficiency for large values of $\mu$. While the spectral efficiency of a noise-limited system increases almost linearly with $\mu(\mathrm{dB})$, it saturates for $\mu>15 \mathrm{~dB}$ in the presence of CCI. Not accounting for the second-tier interferers overestimates the spectral efficiency by $0.5 \mathrm{bits} / \mathrm{sec} / \mathrm{Hz}$ when $\mu=20 \mathrm{~dB}$. Notice again that the simulation and analytical results agree very well.

To understand the relevance of small-scale fading averaged spectral efficiency, Figure 5 plots the spectral efficiency of the system averaged over a window of duration 1 second as a function of time. This is done for a 7-cell, one-sector system with 10 mobile users per cell, and a cell corner SNR of 6 $\mathrm{dB}$. In the simulations, both Rayleigh fading and lognormal shadowing occur and change with time, albeit at different time scales. While Rayleigh fading decorrelates every time a user moves a distance equal to the wavelength, lognormal shadowing has a much longer time correlation coefficient of 0.8 over a time period of 5 seconds. This correlation for lognormal shadowing is generated as per the Gudmundson

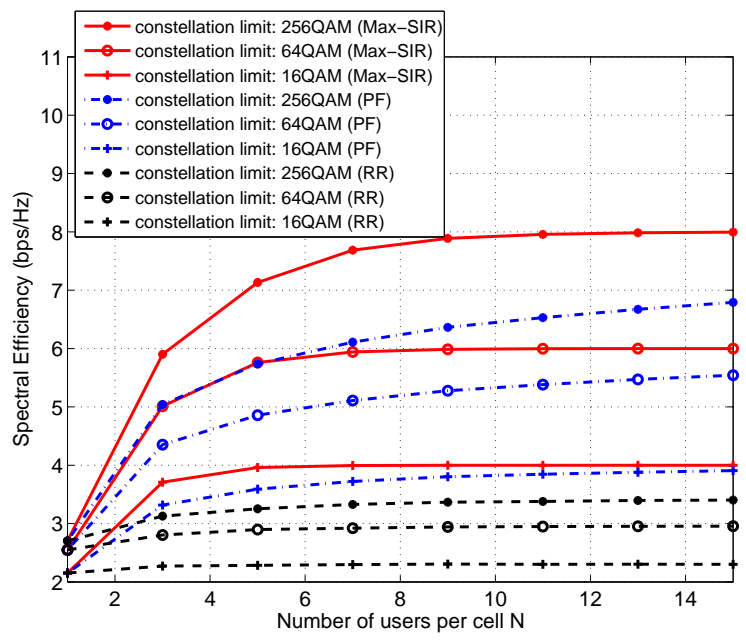

Fig. 7. Composite channels: Effects of constellation size limits on spectral efficiency for RR, PF, and Max-SIR schedulers.

model [27]. We see that the spectral efficiency of the MaxSINR scheduler now changes with time and is sensitive to time variations in lognormal shadowing. On the other hand, the short-term spectral efficiencies of RR and PF schedulers remain relatively flat.

\section{B. Long-Term Spectral Efficiency for Composite Channels}

We now consider spectral efficiency results for Suzuki fading statistics. We first study the case where there is no limit on the modulation constellation size. Figure 6 plots the average spectral efficiency as a function of the number of users per cell when only first-tier interference is considered. The results obtained by using the conventional Fenton-Wilkinson (F-W) lognormal sum approximation [18] and by using the Gaussian$\mathrm{Q}$ function approximation employed are also shown. For a 9-user system, the F-W method overestimates the spectral efficiency by $10.6 \%, 17.3 \%$, and $19.9 \%$ for the RR, PF, and Max-SIR schedulers, respectively. The excellent agreement between the simulation and the new analytical results for all the schedulers demonstrates the accuracy of both the MGF-based lognormal sum approximation and the GaussianQ function approximation employed in the analysis. We also note that the F-W approximation is the major source of errors.

Figure 7 shows the effects of constellation limits on the system spectral efficiency. While the Max-SIR scheduler always outperforms the PF and RR schedulers, limitations on the constellation size undercut its throughput advantage as the Max-SIR scheduler quickly reaches the spectral efficiency cap. While all schedulers benefit from having larger constellation size for adaptive modulation and coding, the Max-SIR scheduler benefits the most and the RR scheduler benefits the least.

\section{CONCLUSIONS}

We derived analytical expressions for the average spectral efficiency of cellular systems for a wide range of schedulers, in exact closed-form when averaging over Rayleigh fading, or in a single integral form when averaging over both short-term fading and long-term shadowing. The spectral efficiency analysis for the Max-SINR/Max-SIR, PF, and RR schedulers was 
sufficiently general to include the effects of Rayleigh fading, lognormal shadowing, non-identical co-channel interference, first- and second-tier interferers, and limited modulation constellation size. Several of the above aspects were not captured by previous works. Sample results show that the impact of sectorization strongly depends on the scheduler - while it is beneficial for RR scheduling, it is detrimental for MaxSINR scheduling. We also found that ignoring second-tier interference overestimates the achievable spectral efficiency by about $0.4 \mathrm{bits} / \mathrm{s} / \mathrm{Hz}$. In general, our results can be used as a benchmark for calibrating the results of system-level simulators, and provide insights into the different factors influencing area spectral efficiency.

\section{A. Proof of Lemma 1 APPENDIX}

Let $\eta=\sum_{m=1}^{M}\left|h_{n m}\right|^{2}+\frac{1}{\rho}$ denote the denominator in the SINR formula given in (4). As the numerator of the SINR expression is an exponential RV, the pdf of the SINR, $\gamma_{n}$, is

$$
f_{\gamma_{n} \mid \alpha}(\gamma)=\int_{0}^{\infty} \frac{\eta}{\alpha_{n 0}} \exp \left(-\frac{\gamma}{\alpha_{n 0}} \eta\right) f_{\eta}(\eta) d \eta
$$

where $f_{\eta}(\eta)$ is the pdf of $\eta$. The form of (33) leads to an alternate and convenient representation of $f_{\gamma_{n} \mid \alpha}(\gamma)$ as:

$$
f_{\gamma_{n} \mid \alpha}(\gamma)=\left.\frac{1}{\alpha_{n 0}} \frac{\partial}{\partial s} M_{\eta}(s)\right|_{s=-\gamma / \alpha_{n 0}}
$$

where $M_{\eta}(s)=\int_{0}^{\infty} e^{\eta s} f(\eta) d \eta=\prod_{m=1}^{M}\left(1-\alpha_{n m} s\right)^{-1} e^{s / \rho}$ is the MGF of $\eta$, and can be easily calculated even though $\eta$ is the sum of not necessarily identical (but independent) chisquared RVs. Combining the above equations results in (8).

\section{B. Proof of Lemma 3}

Based on the subset partition described in Lemma 3 and the $\mathrm{CDF} F_{\gamma_{n}}(\gamma)$ from Corollary 1, the integrand of (7) can be alternatively written as

$$
\frac{1}{1+\gamma}\left[1-F_{\gamma_{n} \mid \alpha}(\gamma)\right]=\exp \left(-\frac{\gamma}{\rho \alpha_{n 0}}\right) \prod_{l=0}^{L_{n}}\left(1+\frac{\alpha_{n}^{(l)}}{\alpha_{n 0}} \gamma\right)^{-m_{n}^{(l)}}
$$

Expanding the product term of (35) in terms of partial fractions, we can rewrite the right hand side of (35) as

$$
\frac{\exp \left(-\frac{\gamma}{\rho \alpha_{n 0}}\right)}{1+\gamma} \sum_{l=1}^{L_{n}} \sum_{i=1}^{m_{n}^{(l)}} \frac{\beta_{i}^{(l)}}{(i-1) !}\left[\frac{\alpha_{n 0}}{\alpha_{n}^{(l)}}\right]^{i-1}\left(1+\frac{\alpha_{n}^{(l)}}{\alpha_{n 0}} \gamma\right)^{-\left(m_{n}^{(l)}-i+1\right)}
$$

where $\alpha_{n}^{(l)}, L_{n}, m_{n}^{(l)}$, and $\beta_{i}^{(l)}$ are defined in the Lemma statement. Substituting the above results in (7) leads to (11).

\section{Proof of Lemma 4}

The CDF of $\gamma_{\max }$ is given by $F_{\gamma_{\max }}(\gamma)=\prod_{n=1}^{N} F_{\gamma_{n} \mid \alpha}(\gamma)$, where $F_{\gamma_{n} \mid \alpha}(\gamma)$ is the CDF of the SINR of the $n$-th user. Substituting the results of Corollary 1 into the expression for $F_{\gamma_{\max }}(\gamma)$ above, we have

$$
F_{\gamma_{\max }}(\gamma)=\prod_{n=1}^{N}\left[1-\prod_{m=1}^{M}\left(1+\frac{\alpha_{n m}}{\alpha_{n 0}} \gamma\right)^{-1} \exp \left(-\frac{\gamma}{\gamma_{0}} \cdot \frac{1}{\alpha_{n 0}}\right)\right] \text {. }
$$

Denote $a_{n}=\prod_{m=1}^{M}\left(1+\frac{\alpha_{n m}}{\alpha_{n 0}} \gamma\right)^{-1} \exp \left(-\frac{\gamma}{\gamma_{0}} \cdot \frac{1}{\alpha_{n 0}}\right)$, then we have $F_{\gamma_{\max }}(\gamma)=\prod_{n=1}^{N}\left(1-a_{n}\right)$, which can be expanded to $F_{\gamma_{\max }}(\gamma)=1+\sum_{n=1}^{N}(-1)^{n} \sum_{k=1}^{\left(\begin{array}{l}N \\ n\end{array}\right)} \prod_{i \in \mathcal{S}_{k}(N, n)} a_{i}$, where $\mathcal{S}_{k}(N, n)$, defined in the Lemma statement, is the $k$-th $\mathrm{n}$ element subset of $\{1, \ldots, N\}$. The result in Lemma 4 immediately follows.

\section{Proof of Theorem 1}

From Lemma 4 and (16), the integrand in (15) can be written as

$$
\frac{\left[1-F_{\gamma_{\max }}(\gamma)\right]}{1+\gamma}=\sum_{n=1}^{N}(-1)^{n-1} \sum_{k=1}^{\left(\begin{array}{c}
N \\
n
\end{array}\right)} e^{-\sigma_{n k} \gamma} \Phi_{n, k}(\gamma)
$$

We then perform a partial fraction expansion of $\Phi_{n, k}(\gamma)$ in (16), use it in (15), and simplify to get the result.

\section{E. Proof of Theorem 2}

Assuming that the $\left\{\varphi_{n}\right\}_{n=1}^{N}$ are identically distributed implies that the CDF of $\varphi_{\max , n}$ is

$$
F_{\varphi_{\max , n}}(x)=F_{\varphi_{n}}^{N-1}(x)=F_{\gamma_{n} \mid \alpha}^{N-1}\left(\bar{\gamma}_{n} x\right)
$$

From (37), we have $C_{\mathrm{PF}}$ = $\sum_{n=1}^{N} \int_{0}^{\infty} C(x) f_{\gamma_{n} \mid \alpha}(x) F_{\gamma_{n} \mid \alpha}^{N-1}(x) d x$, which can be further written as $C_{\mathrm{PF}}=\frac{\log _{2}(e)}{N} \sum_{n=1}^{N} \int_{0}^{\gamma_{T}} \frac{1}{1+x}\left[1-F_{\gamma_{n} \mid \alpha}^{N}(x)\right] d x$. The integrand $\frac{1}{1+x}\left[1-F_{\gamma_{n} \mid \alpha}^{N}(x)\right]$ can be expanded as

$$
\sum_{k=1}^{N}(-1)^{k-1}\left(\begin{array}{c}
N \\
k
\end{array}\right) e^{\frac{k x}{\rho \alpha_{n} 0}} \prod_{l=1}^{L_{n}}\left(1+\frac{\alpha_{n}^{(l)}}{\alpha_{n 0}} x\right)^{-\hat{m}_{n}^{(l)}}
$$

Performing partial fraction expansion of the product term in (38) leads to the result.

\section{F. Proof of Corollary 8}

We have $1-F_{\gamma_{n} \mid \alpha}(x) \leq 1-F_{\gamma_{n} \mid \alpha}^{N}(x)$ because $0 \leq$ $F_{\gamma_{n} \mid \alpha}(x) \leq 1$. Therefore, from (25), $C_{\mathrm{RR}} \leq C_{\mathrm{PF}}$. The equality holds when $F_{\gamma_{n} \mid \alpha}(x)=0$ or 1 . The arithmetic mean geometric mean inequality implies that

$$
\frac{1}{N} \sum_{n=1}^{N} F_{\gamma_{n} \mid \alpha}^{N}(x) \geq \frac{1}{N} \sum_{n=1}^{N} F_{\gamma_{\max }}(x)=\prod_{n=1}^{N} F_{\gamma_{n} \mid \alpha}(x),
$$

This implies that $C_{\mathrm{PF}} \leq C_{\mathrm{MSINR}}$, with equality when $F_{\gamma_{n} \mid \alpha}(x)=$ $F_{\gamma_{m} \mid \alpha}(x), \forall n \neq m$.

\section{REFERENCES}

[1] A. F. Molisch, Wireless Communications. Wiley-IEEE Press, second edition, 2011.

[2] A. Jalali, R. Padovani, and R. Pankaj, "Data throughput of CDMAHDR a high efficiency-high data rate personal communication wireless system," in Proc. VTC, pp. 1854-1858, 2000.

[3] H. Holma and A. Toskala, WCDMA for UMTS. John Wiley, 2000.

[4] S. Catreux, P. Driessen, and L. Greenstein, "Simulation results for an interference-limited multiple-input multiple-output cellular system," IEEE Commun. Lett., vol. 4, pp. 334-336, 2000.

[5] J. Ramiro-Moreno, K. Pedersen, and P. Mogensen, "Network performance of transmit and receive diversity in HSDPA under different packet scheduling strategies," in VTC (Spring), 2003. 
[6] M.-S. Alouini and A. Goldsmith, "Area spectral efficiency of cellular mobile radio systems," IEEE Trans. Veh. Technol., vol. 48, pp. 10471066, 1999.

[7] M. Hasna, M.-S. Alouini, A. Bastami, and E. Ebbini, "Performance analysis of cellular mobile systems with successive co-channel interference cancellation," IEEE Trans. Wireless Commun., vol. 2, pp. 29-40, 2003.

[8] M. Kang, M.-S. Alouini, and L. Yang, "Outage probability and spectrum efficiency of cellular mobile radio systems with smart antennas," IEEE Trans. Commun., vol. 50, pp. 1871-1877, 2002.

[9] IEEE $802.16 \mathrm{~m}$ Evaluation Methodology Document (EMD), IEEE 802.16m-08/004r5, Jan. 2009

[10] H. Dai and H. Poor, "Asymptotic spectral efficiency of multi-cell MIMO systems with frequency-flat fading," IEEE Trans. Sig. Proc., vol. 51, pp. 2976-2988, 2003.

[11] V. Hassel, M. R. Hanssen, and G. E. Øien, "Spectral efficiency and fairness for opportunistic round robin scheduling," in Proc. IEEE Intern. Conf. Commn., pp. 784-789, Jun. 2006.

[12] D. Tse, "Multiuser diversity in wireless networks" http://www.eecs.berkeley.edu/ dtse/ima810.pdf, Apr. 2001.

[13] J.-G. Choi and S. Bahk, "Cell-throughput analysis of the proportional fair scheduler in the single-cell environment," IEEE Trans. Veh. Technol., pp. 766-778, Mar. 2007.

[14] E. Liu and K. Leung, "Expected throughput of the proportional fair scheduling over Rayleigh fading channels," IEEE Commun. Lett., vol. 14, pp. 515-517, June 2010.

[15] H. Fu and D. I. Kim, "Analysis of throughput and fairness with downlink scheduling in WCDMA networks," IEEE Trans. Wireless Commun., vol. 5, pp. 2164-2174, Aug. 2006.

[16] A. Senst, P. Schulz-Rittich, G. Ascheid, and H. Meyr, "On the throughput of proportional fair scheduling with opportunistic beamforming for continuous fading states," in Proc. VTC, 2003.

[17] N. B. Mehta, J. Wu, A. F. Molisch, and J. Zhang, "Approximating a sum of random variables with a lognormal," IEEE Trans. Wireless Commun., vol. 6, pp. 2690-2699, July, 2007.

[18] L. F. Fenton, "The sum of lognormal probability distributions in scatter transmission systems," IRE Trans. Commun. Syst., vol. CS-8, pp. 57-67, 1960.

[19] G. K .Aragiannidis and A. S. Lioumpas "An improved approximation for the Gaussian Q-function," IEEE Commun. Lett., vol. 11, pp. 644-646, Aug. 2007.

[20] "Spatial channel model for multiple input multiple output (MIMO) simulations," Tech. Rep. 25.996, 3rd Generation Partnership Project (3GPP).

[21] G. L. Stüber, Principles of Mobile Communications. Kluwer Academic Publishers, 1996.

[22] I. S. Gradshteyn and I. M. Ryzhik, Table of Integrals, Series and Products. Academic Press, $4^{\text {th }}$ ed., 1980.

[23] S. Catreux, P. Driessen, and L. Greenstein, "Data throughputs using multiple-input multiple-output (MIMO) techniques in a noise-limited cellular environment," IEEE Trans. Wireless Commun., vol. 1, pp. 226235, 2002.

[24] H. Suzuki, "A statistical model for urban radio propagation," IEEE Trans. Commun., vol. 25, pp. 673-677, 1977.

[25] F. Graziosi and F. Santucci, "On SIR fade statistics in Rayleighlognormal channels," in Proc. ICC, pp. 1352-1357, 2002.

[26] J. Wu, N. B. Mehta, A. F. Molisch, and J. Zhang, "Spectral efficiency of channel-aware schedulers in non-identical composite links with interference," in Proc. IEEE Intern. Conf. Commun. ICC'07, pp. 52185223, Jun. 2007.

[27] M. Gudmundson, "Correlation model for shadow fading in mobile radio systems," Elect. Lett., vol. 27, pp. 2145-2146, 1991.

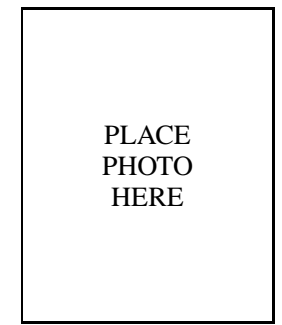

Jingxian Wu (S'02-M'06) received the B.S. degree in electronic engineering from Beijing University of Aeronautics and Astronautics, Beijing, China, in 1998, the M.S. degree in electronic engineering from Tsinghua University, Beijing, China, in 2001, and the Ph.D. degree in electrical engineering from the University of Missouri, Columbia, in 2005.

$\mathrm{He}$ is currently an Assistant Professor with the Department of Electrical Engineering, University of Arkansas, Fayetteville. His research interests mainly focus on wireless communications and wireless networks, including ultralow power communications, cooperative communications, cognitive radio, and cross-layer optimization, etc. He is currently an Editor of the IEEE TRANSACTIONS ON WIRELESS COMMUNICATIONS, and an Associate Editor of the IEEE TRANSACTIONS ON VEHICULAR TECHNOLOGY. He served as a Cochair for the 2012 Wireless Communication Symposium of the IEEE International Conference on Communication, and a Cochair for the 2009 Wireless Communication Symposium of the IEEE Global Telecommunications Conference. Since 2006, he has served as a Technical Program Committee Member for a number of international conferences, including the IEEE Global Telecommunications Conference, the IEEE Wireless Communications and Networking Conference, the IEEE Vehicular Technology Conference, and the IEEE International Conference on Communications.

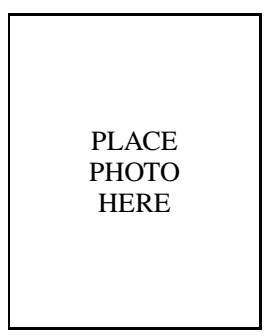

Neelesh B. Mehta (S'98-M'01-SM'06) received his Bachelor of Technology degree in Electronics and Communications Engineering from the Indian Institute of Technology (IIT), Madras, India in 1996, and his M.S. and Ph.D. degrees in Electrical Engineering from the California Institute of Technology, Pasadena, CA, USA in 1997 and 2001, respectively. $\mathrm{He}$ is now an Assistant Professor in the Dept. of Electrical Communication Eng., Indian Institute of Science (IISc), Bangalore, India. Prior to joining IISc, he was a research scientist in the Wireless Systems Research group in AT\&T Laboratories, Middletown, NJ, USA from 2001 to 2002, Broadcom Corp., Matawan, NJ, USA from 2002 to 2003, and Mitsubishi Electric Research Laboratories (MERL), Cambridge, MA, USA from 2003 to 2007.

His research includes work on link adaptation, multiple access protocols, WCDMA downlinks, system-level performance analysis of cellular systems, MIMO and antenna selection, energy harvesting networks, and cooperative communications. He was also actively involved in the Radio Access Network (RAN1) standardization activities in 3GPP from 2003 to 2007. He has served on the TPCs of several conferences. He was a TPC co-chair for WISARD 2010 and 2011 workshops, National Conference on Communications (NCC) 2011, the Transmission Technologies track of VTC 2009 (Fall), and the Frontiers of Networking and Communications symposium of Chinacom 2008. He was the tutorials co-chair for SPCOM 2011. He has co-authored 28 IEEE journal papers, 55 conference papers, and two book chapters, and is a co-inventor in 16 issued US patents. He is an Associate Editor of the IEEE Transactions on Wireless Communications. He is an executive committee member of the IEEE Bangalore Section and the Bangalore chapter of the IEEE Signal Processing Society.

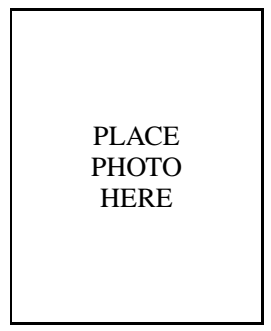


Jin Zhang (S, M, SM, F) received ...

PLACE

PHOTO

HERE 